\title{
MicroRNA-92a-3p inhibits the cell proliferation, migration and invasion of Wilms tumor by targeting NOTCH1
}

\author{
SHIBO ZHU, LIYU ZHANG, ZHANG ZHAO, WEN FU, KAI FU, GUOCHANG LIU and WEI JIA \\ Department of Pediatric Surgery, Guangzhou Women and Children's Medical Center, \\ Guangzhou Medical University, Guangzhou, Guangdong 510623, P.R. China
}

Received October 9, 2017; Accepted May 15, 2018

DOI: $10.3892 /$ or.2018.6458

\begin{abstract}
Dysregulation of miR-92a-3p has been shown to contribute to many tumorigenic processes, and is correlated with tumor progression and prognosis. However, the association between miR-92a-3p and the clinicopathological features of Wilms tumorand its regulatory mechanism remain unknown. In the present study, we demonstrated that miR-92a-3p was downregulated in Wilms tumor tissues and was significantly correlated with the lung metastasis of patients with Wilms tumor. Furthermore, miR-92a-3p mimics suppressed Wilms tumor cell proliferation, migration and invasion by in vitro assays. In addition, miR-92a-3p knockdown promoted tumor progression. Moreover, miR-92a-3p was shown to target directly the 3'-UTR of NOTCH1 mRNA by Dual-Luciferase reporter assays in Wilm's tumor cells. miR-92a-3p mimics decreased the expression of mRNA and protein of NOTCH1. miR-92a-3p inhibitor enhanced NOTCH1 expression by using western blotting and qPCR. In Wilms tumor tissues, NOTCH1 was highly expressed when compared with that in adjacent non-tumor tissues. NOTCH1 expression was found to be negatively correlated with miR-92a-3p in tumor tissues. Knockdown of NOTCH1 expression reversed the promotive effect of miR-92a-3p inhibitor on the cell proliferation, migration and invasion in Wilms tumor. In conclusion, miR-92a-3p blocks the progression of Wilms tumor by targeting NOTCH1.
\end{abstract}

\section{Introduction}

Wilms tumor is the most common pediatric renal tumor with a prevalence of $\sim 1$ in 10,000 children (1). Although combination therapy has improved the prognosis for most patients, $\sim 10 \%$ of

Correspondence to: Dr Guochang Liu or Dr Wei Jia, Department of Pediatric Surgery, Guangzhou Women and Children's Medical Center, Guangzhou Medical University, 9 Jinsui Road, Tianhe, Guangzhou, Guangdong 510623, P.R. China

E-mail: starbless2003@126.com

E-mail: jiawei198044@hotmail.com

Key words: Wilms tumor, miR-92a-3p, NOTCH1, migration, invasion patients with Wilms tumors experience poor survival due to metastasis and recurrence (2-5). Hence, it is essential to elucidate the molecular mechanism underlying the tumorigenesis and metastasis of Wilms tumors, which could provide predictive and therapeutic targets for this childhood disease.

MicroRNAs (miRNAs) are a class of single-stranded, highly conserved small non-coding RNAs that regulate gene expression at the post-transcriptional level by binding to the 3'-unstranslated region (UTR) of target mRNAs, resulting in mRNA silencing or degradation (6-8). In tumors, various studies have confirmed that miRNAs function as oncogenes or tumor suppressors which regulate tumor initiation, progression and prognosis (9-11). The expression of miRNAs has been shown to be stable and an excellent marker for the early diagnosis of tumors (12).

miR-92a-3p is a member of the miR-17-92 family, which plays a critical role in modulating cell viability, apoptosis and metastasis of tumor cells $(13,14)$. In glioma, miR-92a-3p was found to exert various effects on tumor stem-like cells by targeting the Notch-1/Akt pathway (15). In colorectal adenocarcinoma, the expression level of miR-92a-3p was able to predict the prognosis of patients (16). However, the expression level, clinicopathological and biological functions of miR-92a-3p in Wilms tumor and its underlying molecular mechanisms remain unclear. In the present study, we aimed to investigate the predicted miRNAs and the related molecular mechanisms in Wilms tumor.

\section{Materials and methods}

Microarray data. The gene expression profiles of GSE50505 (https://www.ncbi.nlm.nih.gov/geo/query/acc.cgi?acc=GSE50505), GSE57370 (https://www.ncbi.nlm.nih.gov/geo/query/acc. cgi?acc=GSE57370) and GSE17342 (https://www.ncbi.nlm.nih. gov/geo/query/acc.cgi?acc=GSE17342) were downloaded from the GEO database. GSE50505, which was based on GPL17667 platform (Luminex Homo sapiens bead-based microRNA profiling platform), was submitted by Liu et al. The dataset contained 28 samples, including 20 Wilms tumor samples and 3 normal kidney samples. GSE17342 was based on GPL8367 platform (LC_MRA-1001_miRHuman_10.0_070802), including 2 Wilms tumor samples and 2 normal kidney samples. GSE57370 was based on GPL16770 platform (Agilent-031181 Unrestricted_Human_miRNA_V16.0_Microarray), including 62 Wilms tumor samples and 4 normal kidney samples. 
Patients and tissue samples. Wilms tumor tissues and the corresponding adjacent non-tumor tissues were obtained from 68 patients who had Wilms tumors and had undergone surgery at the Women and Chidren's Hospital of Guangzhou Medical University (Guangzhou, China) between July 2012 and July 2017. The age and sex distribution of WT patients are shown in Table I. Adjacent non-tumor tissues were obtained $3 \mathrm{~cm}$ away from the tumor, and the lack of tumor cell infiltration was verified by pathological examination. All tissue samples were frozen in liquid nitrogen and stored at $-80^{\circ} \mathrm{C}$. All patients had not received chemotherapy or radiotherapy before the surgery. Informed consent was obtained from each patient, and the study protocol and consent procedures were approved by the Ethics Committee of Guangzhou Medical University (Guangzhou, China).

Primary cell line and culture. The fresh tumor tissues were sliced into $0.1 \mathrm{~cm}^{3}$ pieces and washed with phosphate-buffered saline (PBS; Gibco; Thermo Fisher Scientific, Inc., Waltham, MA, USA). The tissues were then incubated overnight with $2 \mathrm{U} / \mathrm{ml}$ dispase (Gibco; Thermo Fisher Scientific, Inc.) at $4^{\circ} \mathrm{C}$ on a stirrer at $100 \mathrm{rpm}$, followed by digestion with $160 \mu \mathrm{g} / \mathrm{ml}$ of collagenase A (Sigma-Aldrich; Merck KGaA, Darmstadt, Germany) at $37^{\circ} \mathrm{C}$ for $3 \mathrm{~h}$. Thecollected and digested cells were cultured in Dulbecco's modified Eagle's medium (DMEM; Invitrogen; Thermo Fisher Scientific, Inc.) with $10 \%$ fetal bovine serum (FBS; Invitrogen; Thermo Fisher Scientific, Inc.) until the cells had grown in a confluent monolayer at $37^{\circ} \mathrm{C}$ in a humidified chamber supplemented with $5 \% \mathrm{CO}_{2}$. A maintenance culture was carried out in a $25-\mathrm{ml}$ flask with DMEM supplemented with $10 \%$ FBS and 100 units/ml of streptomycin and $100 \mu \mathrm{g} / \mathrm{ml}$ penicillin (both from Gibco; Thermo Fisher Scientific, Inc.). The culture medium was replaced every 2 days and the cells were propagated every 3 days. For cryopreservation, the cells were frozen in DMEM containing 10\% dimethyl sulfoxide (DMSO; Sigma-Aldrich; Merck $\mathrm{KGaA}$ ) and $90 \%$ FBS and stored in liquid nitrogen.

Transient transfection. The miR-92a-3p mimic, miR-92a-3p inhibitor and corresponding negative control (miR-NC) were purchased from Shanghai GenePharma Co., Ltd. (Shanghai, China). The siRNA against NOTCH1 (UGGCGGGAA GUGUGAAGCG) and its negative control were provided by Takara Bio Inc. (Otsu, Japan). These molecular products were transfected into cells using Lipofectamine 2000 (Invitrogen; Thermo Fisher Scientific, Inc.) for the various experiments according to the manufacturer's instructions. Briefly, the final concentration of these products was $50 \mathrm{nM}$ and the cells were harvested for subsequent experiments at $24 \mathrm{~h}$ after transfection. Each experiment was repeated three times each in triplicates.

RNA extraction and quantitative real-time PCR. Total RNA was isolated from the Wilms tumor tissues, matched adjacent normal tissues and WT cells using TRIzol ${ }^{\circledR}$ reagent (Invitrogen; Thermo Fisher Scientific, Inc.). Briefly, all samples were treated with TRIzol followed by chloroform. The mixture was centrifuged at $14,000 \mathrm{rpm}$ for $10 \mathrm{~min}$ at $4^{\circ} \mathrm{C}$ and $700 \mu 175 \%$ ethanol was added to the aqueous layer. Finally, the purified RNA was diluted with $30 \mu \mathrm{l}$ of RNase-free water. cDNA synthesis was performed with $2 \mu \mathrm{g}$ total RNA using the PrimeScript ${ }^{\mathrm{TM}}$ RT reagent kit with gDNA Eraser (Takara Bio, Inc., Otsu, Japan) for the next qPCR with Mir-X miRNA First-Strand Synthesis kit (Takara Bio, Inc.) for microRNA according to the manufacturers' instructions. The primers sequences of NOTCH1 were F, TGCCAGACCAAC ATCAAC and R, CTCATAGTCCTCGGATTGC (Takara Biotechnology, Co., Ltd., Dalian, China). The sequences of miR-92a-3p were F: GGGGCAGTTATTGCACTTGTC and R:General reverse primer for microRNA is purchased from RiboBio Co. Ltd. (Guangzhou, China). The sequences of GAPDH were F: GCACCGTCAAGGCTGAGAAC and R: TGGTGAAGACGCCAGTGGA (Takara Biotechnology). A qPCR was performed using the SYBR Premix Ex Taq II kit (Takara Bio) and the Applied Biosystems 7500 Fluorescent Quantitative PCR system (Applied Biosystems; Thermo Fisher Scientific, Inc.). The mixtures were incubated at $95^{\circ} \mathrm{C}$ for $30 \mathrm{sec}$, followed by 40 amplification cycles of $95^{\circ} \mathrm{C}$ for $5 \mathrm{sec}$ and $60^{\circ} \mathrm{C}$ for $34 \mathrm{sec}$. The comparative cycle threshold method was used to quantify the relative expression levels of mRNA and microRNA. Expression levels of the housekeeping gene GAPDH and U6 were used to normalize the expression levels of the genes-of-interest, respectively. The relative mRNA levels were calculated based on the $\mathrm{Ct}$ values and normalized using the relative housekeeping gene expression.

Cell proliferation assay. In vitro cell proliferation was measured using the 3-(4,5-dimethylthiazol-2-yl)-2,5diphenyltetrazolium bromide (MTT) method following the manufacturer's instructions (Nanjing KeyGen Biotech, Nanjing, China). Briefly, the transfected cells were seeded into 96 -well plates $\left(2 \times 10^{3}\right.$ cells/well) and cultured for 5 days. The MTT solution (formazan in DMSO) was added to each well at the indicated time-points (1, 2, 3, 4 and 5 days) and incubated at $37^{\circ} \mathrm{C}$ for $4 \mathrm{~h}$. The optical density value (OD) of each well was measured at $450 \mathrm{~nm}$ using a microplate spectrophotometer (BioTek Instruments, Inc., Winooski, VT, USA).

Cell colony formation assay. The transfected cells were seeded into 6-well plates at a density of 100 cells/well. After culture for 10 days, the colonies were washed with PBS, fixed with $4 \%$ paraformaldehyde and stained with $1 \%$ crystal violet. The colonies were imaged and counted in five randomly selected fields under a light microscope (Olympus Corp., Tokyo, Japan).

Wound healing assay. Briefly, cells $\left(1 \times 10^{5}\right)$ were seeded in 6-well plates and incubated overnight. A wound was created with a $10-\mu \mathrm{m}$ pipette tip and images were obtained under a light microscope (Olympus Corp.). The wound gaps were measured per time-point.

Transwell assay. The assays were carried out in Transwell chambers (8- $\mu \mathrm{m}$ pore size) (Corning, Inc., Corning, NY, USA). Matrigel $^{\mathrm{TM}}$ Matrix (BD Biosciences, San Jose, CA, USA) was diluted 1:7 using serum-free basal medium and $50 \mu 1$ Matrigel Matrix dilution was added to the upper chamber of the Transwell inserts. Moreover, $100 \mu \mathrm{l}$ transfected cell $\left(2 \times 10^{5} / \mathrm{ml}\right)$ suspensions were seeded in the upper chambers precoated with Matrigel Matrix dilution in 24-well plates and cultured in serum-free basal medium. A total of $500 \mu 1$ medium with $10 \%$ FBS was also added to the lower chambers. After $24 \mathrm{~h}$, cells 
in the upper chambers were removed using cotton swabs. The inserts was washed three times with PBS, and cells that invaded to the bottom surface of the insert were fixed with $4 \%$ paraformaldehyde and stained using $1 \%$ crystal violet. The invading cells were countedunder a Leica DMI4000B microscope (Leica Microsystems, Heidelberg, Germany) from randomly selected five fields and photomicrographs were captured.

Luciferase reporter assay. The wild-type (WT) or mutant-type (MUT) seed region at the 3'UTR of NOTCH1 was synthesized and cloned into the downstream region of a firefly luciferase cassette in the pGL3-promoter vector (Promega Corporation, Madison, WI, USA) according to the manufacturer's instructions. The cells were cotransfected with vectors carrying the WT 3'UTR or MUT 3'UTR NOTCH1 and miR-92a-3p mimic or miR-NC by using Lipofectamine 2000 reagent according to the manufacturer's instructions. After a 48-h transfection, the cells were harvested to detect luciferase activity by using the Dual-Luciferase assay (Promega Corporation).

Western blotting assay. Total proteins were extracted from cells or tissues with RIPA buffer (10 mM Tris-HCl, $\mathrm{pH} 7.4$, $1 \%$ Triton $\mathrm{X}-100,0.1 \%$ SDS, $1 \% \mathrm{NP}-40$ and $1 \mathrm{mM} \mathrm{MgCl}$ ) containing protease inhibitors. The total protein concentration was determined using a BCA Protein Assay kit (Nanjiing KeyGen Biotech). A total of $30 \mathrm{mg}$ of protein was separated on a $10 \%$ SDS-polyacrylamide gel and then transferred onto polyvinylidene fluoride (PDVF) membranes (Sigma-Aldrich; Merck KGaA). The membranes were blocked with $5 \%$ milk and then incubated with primary antibodies against NOTCH1 (rabbit IgG, 1:1,000; cat. no. 3608S ) and GAPDH (rabbit IgG, 1:1,000; cat. no. 5174) overnight at $4^{\circ} \mathrm{C}$. On the second day, the blots were washed with PBST and incubated with secondary antibodies (anti-rabbit IgG, 1:2,000; cat. no. 7074) for $2 \mathrm{~h}$ at room temperature. The antibodies were purchased from Cell Signaling Technology, Inc. (Danvers, MA, USA). The protein band was visualized by chemiluminescence imaging system (ChemiDoc Touch; Bio-Rad Laboratories, Hercules, CA, USA). GAPDH was used as an internal control.

Statistical analysis. For comparisons, two-tailed Student's t-test, Wilcoxon rank-test, Fisher's exact test, one-way analysis of variance (ANOVA) test and the Kruskal-Wallis test were performed. Overall survival (OS) was calculated and multivariate Cox's proportional harzards model was performed to determine the independent factors. Survival curves were performed by Kaplan-Meier's method and calculated by log-rank test. For correlation, Spearman's and Pearson's correlation were used. Statistical analyses were conducted using SPSS 13.0 software (SPSS, Inc., Chicago, IL, USA) with a two-sided significance level of $\mathrm{P}<0.05$.

\section{Results}

miR-92a-3p is downregulated in Wilms tumors. To reveal the expression of miRNAs in Wilms tumor, we downloaded the microarray chips concerning the miRNA gene expression profiles of GSE50505, GSE57370 and GSE17342. We chose the genes with $\mathrm{P}<0.05$ and fold control (FC) 1.5 as criteria. After analysis with InteractiVenn, we obtained a common
Table I. Relationship between miR-92a-3p and the clinicopathological features of the Wilms tumor cases.

$$
\text { miR-92a-3p expression }
$$

\begin{tabular}{|c|c|c|c|}
\hline \multirow[b]{2}{*}{ Characteristics } & & \multirow[b]{2}{*}{ P-value } \\
\hline & High (\%) & Low $(\%)$ & \\
\hline Sex & & & 0.061 \\
\hline Male & 38.2 & 16.2 & \\
\hline Female & 30.9 & 14.7 & \\
\hline Age (years) & & & 0.832 \\
\hline$<4$ & 41.2 & 11.8 & \\
\hline$\geq 4$ & 27.9 & 19.1 & \\
\hline Tumor size $(\mathrm{cm})$ & & & 0.710 \\
\hline$<10$ & 51.5 & 16.2 & \\
\hline$\geq 10$ & 17.6 & 14.7 & \\
\hline Histologic type & & & 0.353 \\
\hline Triphasic & 11.8 & 5.9 & \\
\hline Blastemal & 13.2 & 4.4 & \\
\hline Stromal & 17.6 & 8.8 & \\
\hline Epithelial & 16.2 & 10.3 & \\
\hline Others & 5.9 & 4.4 & \\
\hline Lung metastasis & & & 0.042 \\
\hline No & 23.5 & 35.5 & \\
\hline Yes & 8.8 & 32.4 & \\
\hline Survival status & & & 0.016 \\
\hline Alive & 38.2 & 26.5 & \\
\hline Deceased & 7.4 & 33.8 & \\
\hline
\end{tabular}

P-values in bold print indicate significant difference.

gene miR-92a-3p (Fig. 1A). To reveal the role of miR-92a-3p in Wilms tumor, RT-qPCR was performed to examine the expression levels of miR-92a-3p in tumor samples and adjacent non-tumor tissues of $68 \mathrm{Wilms}$ tumor patients. As revealed in Fig. 1B, miR-92a-3p was frequently downregulated in Wilms tumor, compared with that in the adjacent tissues $(\mathrm{P}<0.001)$. To further investigate the clinical significance of miR-92a-3p expression in Wilms tumor, we tested the expression level of miR-92a-3p in patients with or without metastasis and found that miR-92a-3p expression was lower in patients with metastasis than patients without metastasis $(\mathrm{P}<0.01)$ (Fig. 1C). We divided the 68 patients into two groups according to the median value of miR-92a-3p expression in the Wilms tumors: high miR-92a-3p expression group and low miR-92a-3p expression group (Table I). In addition, Kaplan-Meier's analysis (Fig. 1D) indicated that Wilms tumor patients with low miR-92a-3p expression exhibited poorer overall survival $(\mathrm{P}<0.05)$.

miR-92a-3p inhibits Wilms tumor cell proliferation and colony formation. To examine the effect of miR-92a-3p on Wilms tumor growth, we obtained the primary cells from a Wilms tumor (Fig. 2A). The Wilms tumor cells were then transfected with the miR-92a-3p mimic, miR-NC and inhibitor. miR-92a-3p expression was comfirmed by qPCR (Fig. 2B). The MTT assay (Fig. 2C) indicated that WT cells with higher miR-92a-3p expression exhibited reduced proliferation 

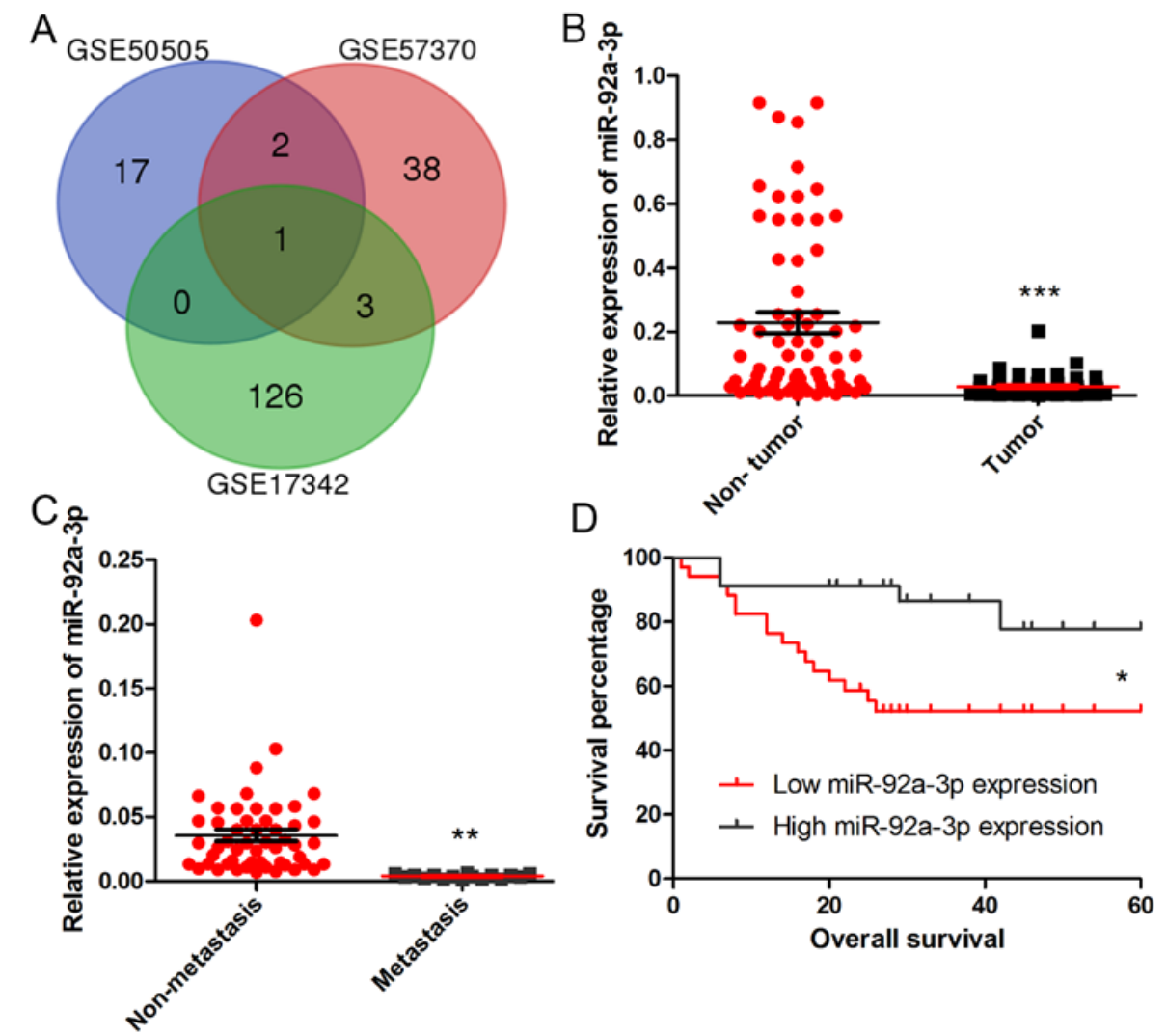

Figure 1. miR-92a-3p is downregulated in Wilms tumors. (A) We obtained a common gene miR-92a-3p after analysis with the results of the microarray chips (B) miR-92a-3p was downregulated in Wilms tumor tissues when compared with that in adjacent non-tumor tissues. (C) The expression of miR-92a-3p was lower in patients with metastasis than with non-metastasis. (D) Kaplan-Meier's survival curves indicated that the Wilms tumor patients with high miR-92a-3p expression had a better overall survival when compared with patients with low miR-92a-3p expression. Data are shown as mean $\pm \mathrm{SD}$. ${ }^{*} \mathrm{P}<0.05,{ }^{* * *} \mathrm{P}<0.01$, ${ }^{* * *} \mathrm{P}<0.001$.

compared to the cells transfected with miR-NC. In contrast, the Wilms tumor cells with low miR-92a-3p expression exhibited increased proliferation $(\mathrm{P}<0.05)$. The colony formation assay (Fig. 2D and E) demonstrated that Wilms tumor cells transfected with the miR-92a-3p mimic developed significantly lower rates of colony formation when compared with the cells transfected with miR-NC. Additionaly, Wilms tumor cells transfected with the miR-92a-3p inhibitor had a higher rate of colony formation $(\mathrm{P}<0.05)$. These results suggest that miR-92a-3p inhibitedthe proliferation and colony formation of Wilms tumor cells.

miR-92a-3p inhibits Wilms tumor cell migration and invasion. To measure the effect of miR-92a-3p on the migratory and invasive capacities of theWilms tumor cells, we used Matrigel-coated Transwell experiments and wound healing assays. The results revealed a significant decrease in the wound-healing distance in the miR-92a-3p mimic-transfected WT cells after $24 \mathrm{~h}$. Meanwhile, the wound-healing distance of the miR-92a-3p inhibitor-transfected Wilms tumor cells was more extensive when compared with the miR-NC cells (Fig. 3A and B). We observed that the miR-92a-3p mimic significantly decreased the invasiveness of theWilms tumor cells through Matrigel. The miR-92a-3p inhibitor increased the invasion potential of the Wilms tumor cells (Fig. 3C and D). These results demonstrated that miR-92a-3p inhibits the potential of Wilms tumor cells in terms of migration and invasion.
NOTCH1 is a direct target of miR-92a-3p in Wilms tumor. To explore the molecular mechanism by which miR-92a-3p functions in Wilms tumor, we used bioinformatic prediction software (TargetScan) to determine the potential target. We identified that miR-92a-3p was able to bind the 3'-UTR of NOTCH1 (Fig. 4A). To further confirm this binding, we performed a luciferase assay and demonstrated that miR-92a-3p dramatically inhibited the luferase activity of the wild-type (WT) 3'-UTR but not of the mutant-type (Mut) 3'-UTR and blank vector of NOTCH1 (Fig. 4B). Moreover, miR-92a-3p mimic significantly inhibited mRNA and protein expression of NOTCH1 and the inhibitor promoted the NOTCH1 expression (Fig. 4C and D).

NOTCH1 knockdown rescued the effect of miR-92a-3p inhibitor on Wilms tumor cells. To further determine whether NOTCH1 is a functional target of miR-92a-3p in Wilms tumor, we performed a rescue experiment. NOTCH1 siRNA reduced the promotive effects of the miR-92a-3p inhibitor on proliferation, migration and invasion of Wilms tumor cells (Fig. 5A-D). To explore the relationship between miR-92a-3p and NOCTH1 in Wilms tumortissues, RT-qPCR was performed to test the expression of NOTCH1. As shown in Fig. 5E, Wilms tumor tissues had significantly higher levels of NOTCH1 mRNA than those of adjacent non-tumor tissues. Moreover, NOTCH1 mRNA had an inverse correlation with miR-92a-3p expression in Wilms tumor tissues (Fig. 5F). 

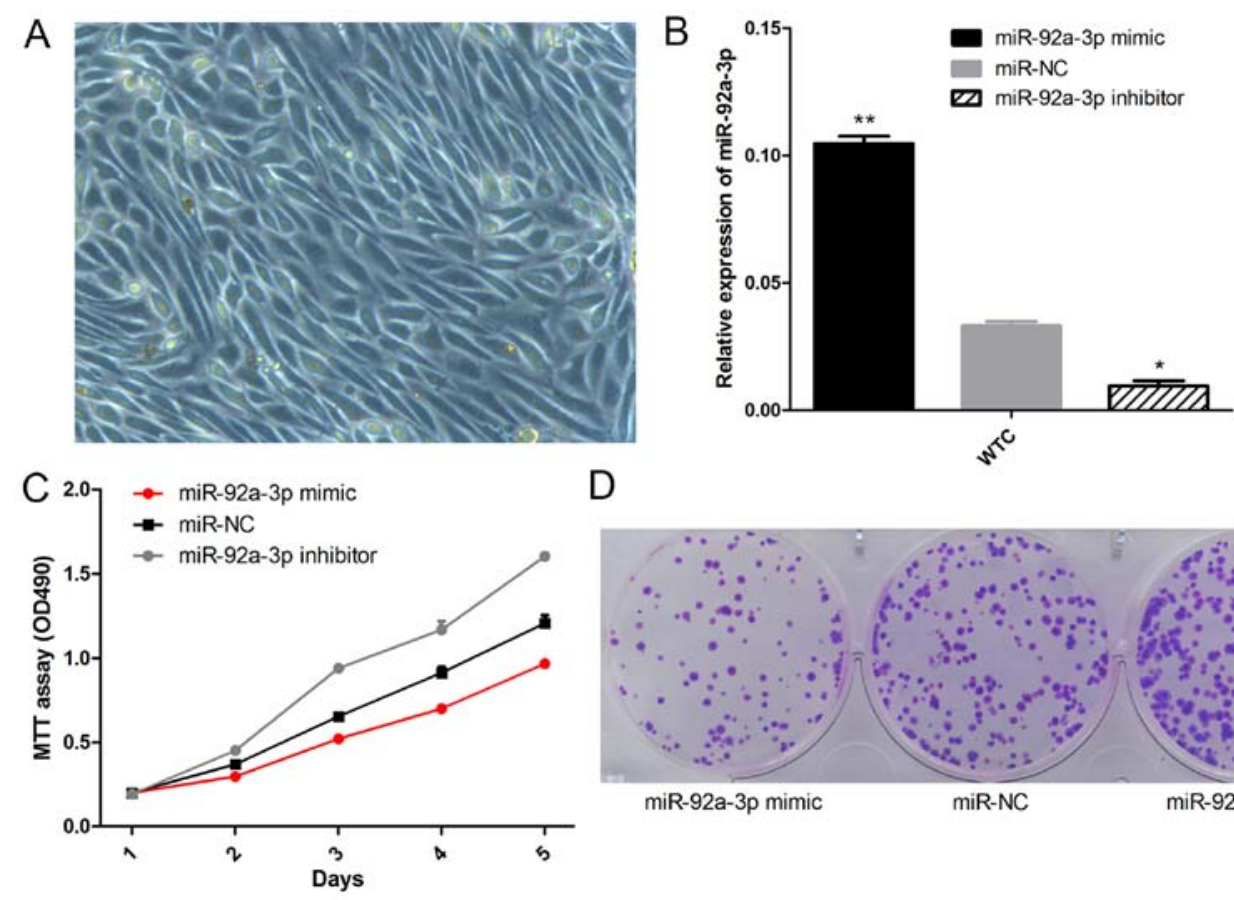

D
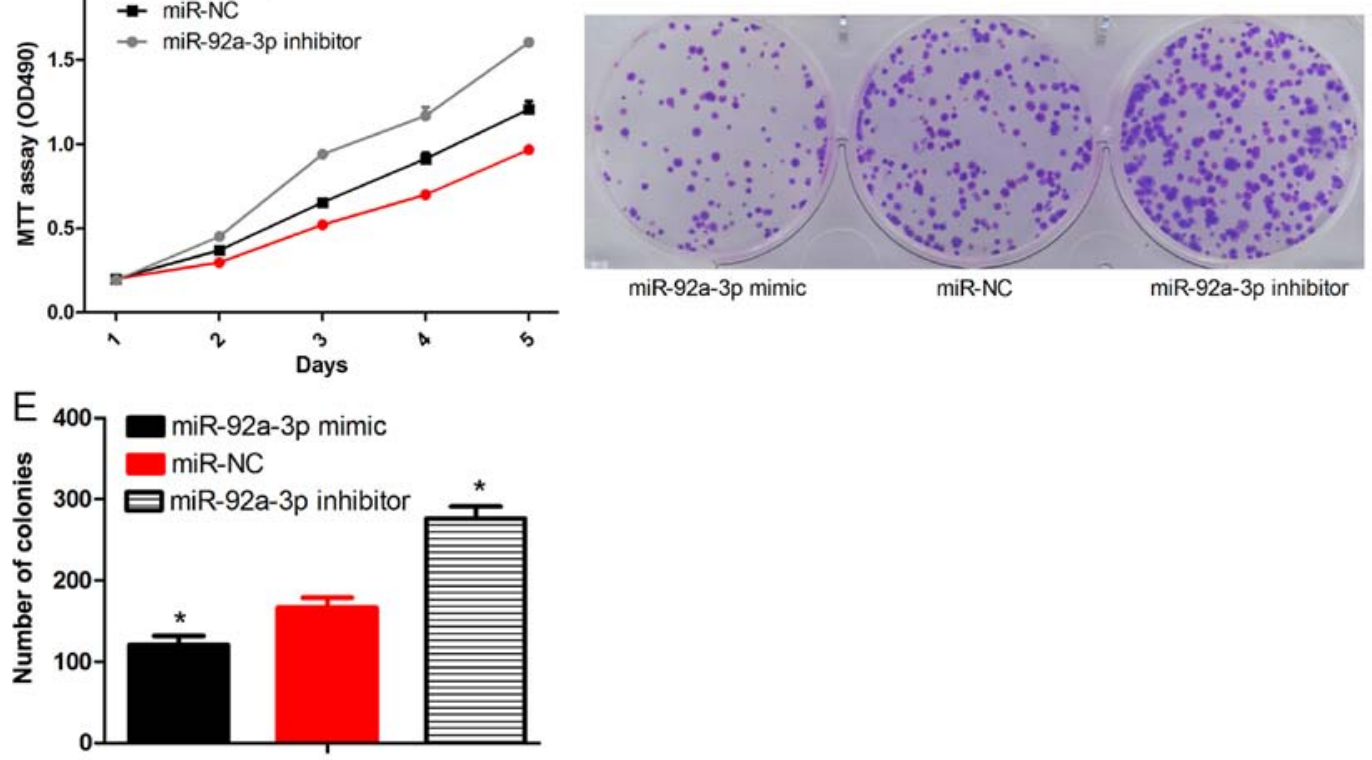

Figure 2. miR-92a-3p inhibits Wilms tumor cell proliferation and colony formation. (A) The tumor cell line WTC from a Wilms tumor patient. (B) The expression of miR-92a-3p was detected after miR-92a-3p mimic and inhibitor transfection of the WTC. (C) MTT assays showed that the proliferation capacity of WTC increased after miR-92a-3p inhibitor transfection, and it decreased after mimic transfection. (D and E) The colony formation capacity of WTC was suppressed after miR-92a-3p mimics, and it was promoted after inhibitor transfection. ${ }^{*} \mathrm{P}<0.05,{ }^{* *} \mathrm{P}<0.01$.

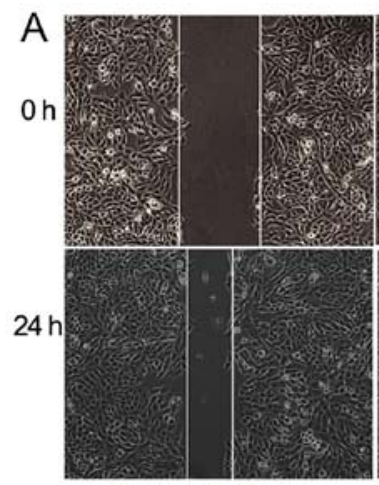

$\mathrm{C}$

$$
\text { miR-92a-3p mimic }
$$

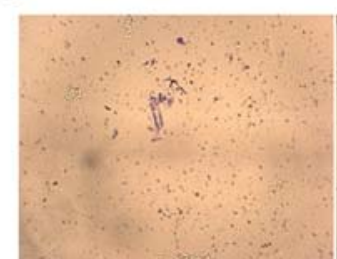

miR-92a-3p mimic

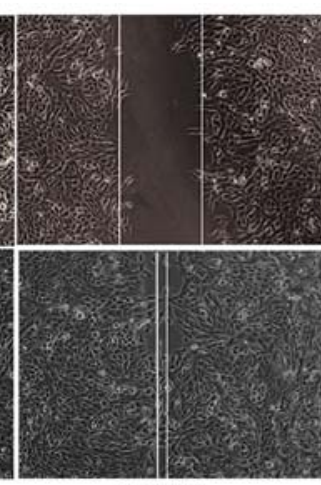

miR-NC

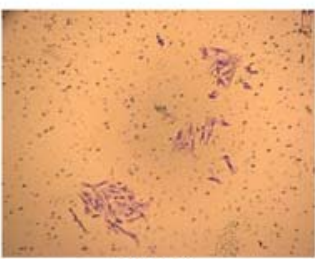

miR-NC

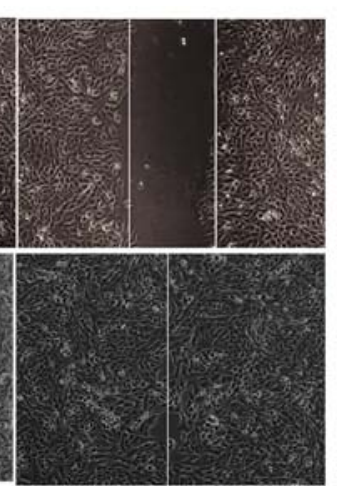

miR-92a-3p inhibitor

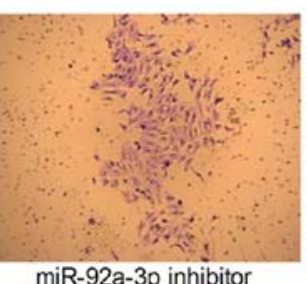

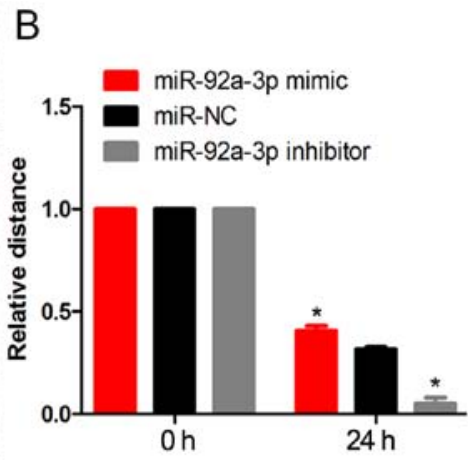

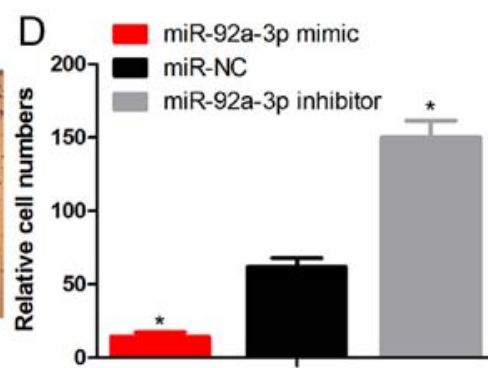

Figure 3. miR-92a-3p inhibits Wilms tumor cell migration and invasion. (A and B) The distance of WTC cells after miR-92a-3p mimic are greater than negative control cells, and miR-92a-3p inhibitor transfection reduced the distance significantly. (C and D) miR-92a-3p mimics significantly reduced the invasion potential of WTC cells. The miR-92a-3p inhibitors increased the invasion potential of these cells. ${ }^{*} \mathrm{P}<0.05,{ }^{* *} \mathrm{P}<0.01$. 


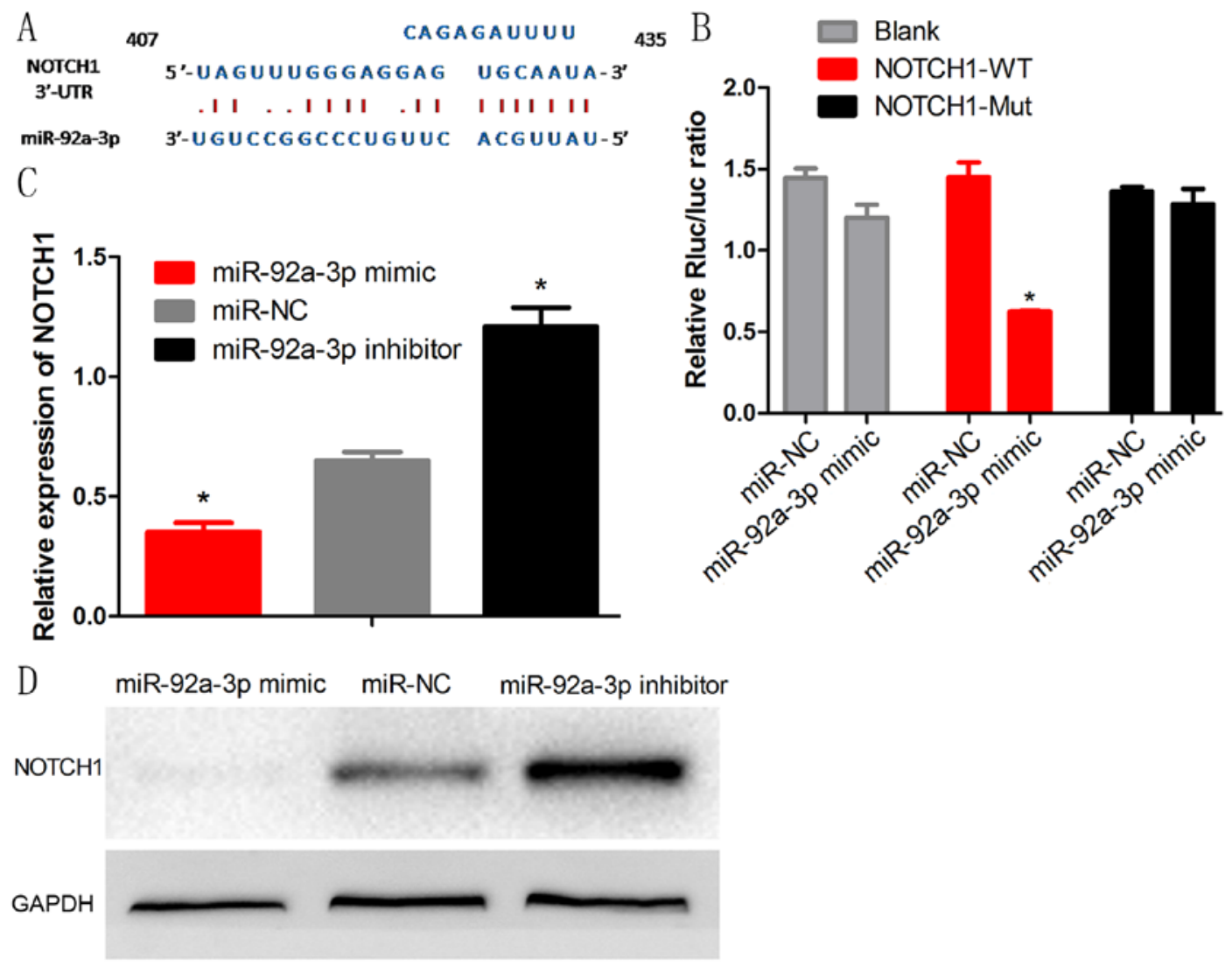

Figure 4. NOTCH1 is a direct target of miR-92a-3p in Wilms tumor. (A) The TargetScan and MicroRNA predicted the align sequences between the NOTCH1 3'-UTR and miR-92a-3p. (B) miR-92a-3p mimic reduced the luciferase activities of the WT reporter vector but not empty vector or mutant reporter vector. (C) The expression of NOTCH1 after miR-92a-3p mimic was significantly decreased and increased after inhibitor transfection. (D) The NOTCH1 protein expression after miR-92a-3p mimic and inhibitor transfection. ${ }^{\mathrm{P}}<0.05$.

\section{Discussion}

A series of microarray chips have been used to detect the miRNA expression of Wilms tumors and these studies reported the abnormal expression levels of various miRNAs in Wilms tumor, such as the upregulated genes, miR-378 and miR-18b and the downregulated genes, miR-193a-5p and miR-199a-5p $(5,17)$. However, studiesconcerning the cliniopathological and biological mechanisms concerning miRNAs in Wilms tumors are sparse. In the present study, we extracted the data from microarray profiles of GSE50505, GSE57370 and GSE17342, including thousands of miRNA genes in the human genome simultaneously, which has been widely used to predict the potential therapeutic targets for tumors. Notably, we identified a common downregulated gene miR-92a-3p in Wilms tumor. The present study further confirmed the low expression of miR-92a-3p in Wilms tumor and we also found that overexpression of miR-92a-3p inhibited the proliferation, migration and invasion of Wilms tumor cells. In addition, miR-92a-3p knockdown showed contrary results. These results indicate that miR-92a-3p may serve as a tumor suppressor.

NOTCH1 is a member of the Notch family, the evolutionarily conserved family of transmembrane receptors, which regulate cell fate, stem cell self-renewal and differentiation during development $(18,19)$. Recently, Notch1 was reported to take part in diverse tumor processes including cell proliferation, apoptosis, and cancer metastasis and angiogenesis in various types of cancer $(20,21)$. In addition, in kidney development, Notch receptors were reported to regulate mesangial cell specification, proliferation or survival (22). These results suggest that NOTCH family receptors may play pivotal roles in Wilms tumor. In the present study, we found that the expression of NOTCH1 was downregulated by miR-92a-3p mimic, and NOTCH1 was upregulated by the miR-92a-3p inhibitor. Moreover, the functions of miR-92a-3p inhibitor on Wilms tumor were reversed by NOTCH1 knockdown. The expression of NOTCH1 and miR-92a-3p had an obvious negative correlation in Wilms tumor. The results above suggest that NOTCH1 is a direct target of miR-92a-3p and miR-92a-3p inhibits the proliferation, migration and invasion of Wilms tumor by targeting NOTCH1.

In conclusion, the present study demonstrated that miR-92a-3p was downregulated inWilms tumor tissues and significantly correlated with the lung metastasis of patients. Furthermore, miR-92a-3p mimics suppressed Wilms tumor cell proliferation, migration and invasion. Additionally, miR-92a-3p knockdown promoted the progression. Moreover, NOTCH1 is a direct target of miR-92a-3p and miR-92a-3p inhibits tumor progression by targeting NOTCH1. Knockdown of NOTCH1 expression reversed the promotive effect of the miR-92a-3p inhibitor on Wilms tumor progression. In conclusion, miR-92a-3p blocks the progression of Wilms tumor by targeting NOTCH1 (Fig. 6). 

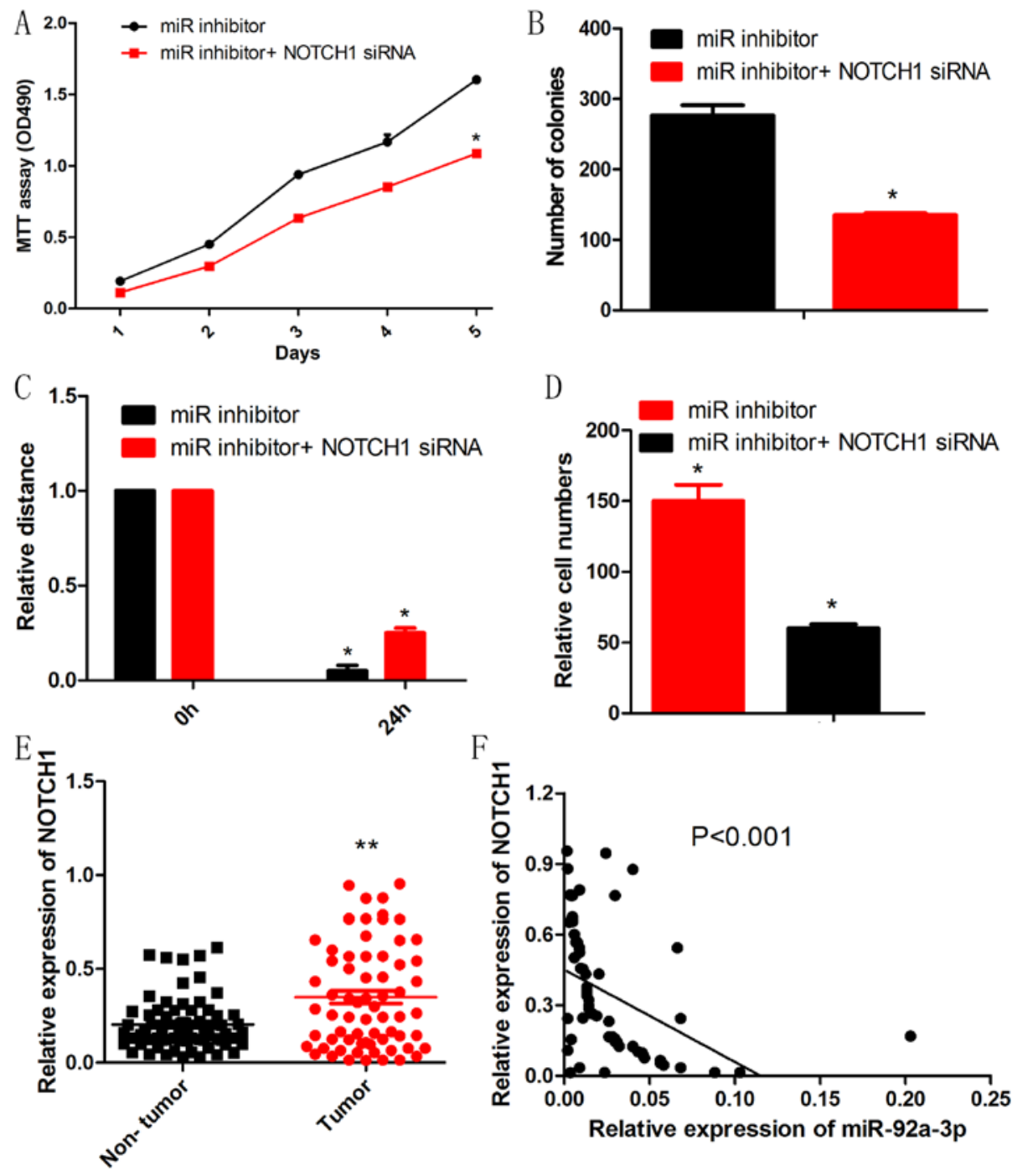

Figure 5. NOTCH1 knockdown rescued the effect of the miR-92a-3p inhibitor on Wilms tumor cells. (A) MTT assays showed that NOTCH1 knockdown reduced the proliferation ability of WTC cells. (B) Colony formation assays showed that NOTCH1 knockdown decreased the colony formation ability of the WTC cells. (C) Wound healing assays showed that NOTCH1 knockdown suppressed the invasive ability of the WTC cells. (D) Transwell assays showed that NOTCH1 knockdown suppressed the migration ability of the WTC cells. (E) The expression of NOTCH1 was higher in the tumor tissues than that noted in the adjacent non-tumor. (F) The expression of NOTCH1 and miR-92a-3p was significantly inversely correlated in Wilms tumor patient tissues. ${ }^{*} \mathrm{P}<0.05,{ }^{* *} \mathrm{P}<0.01$.

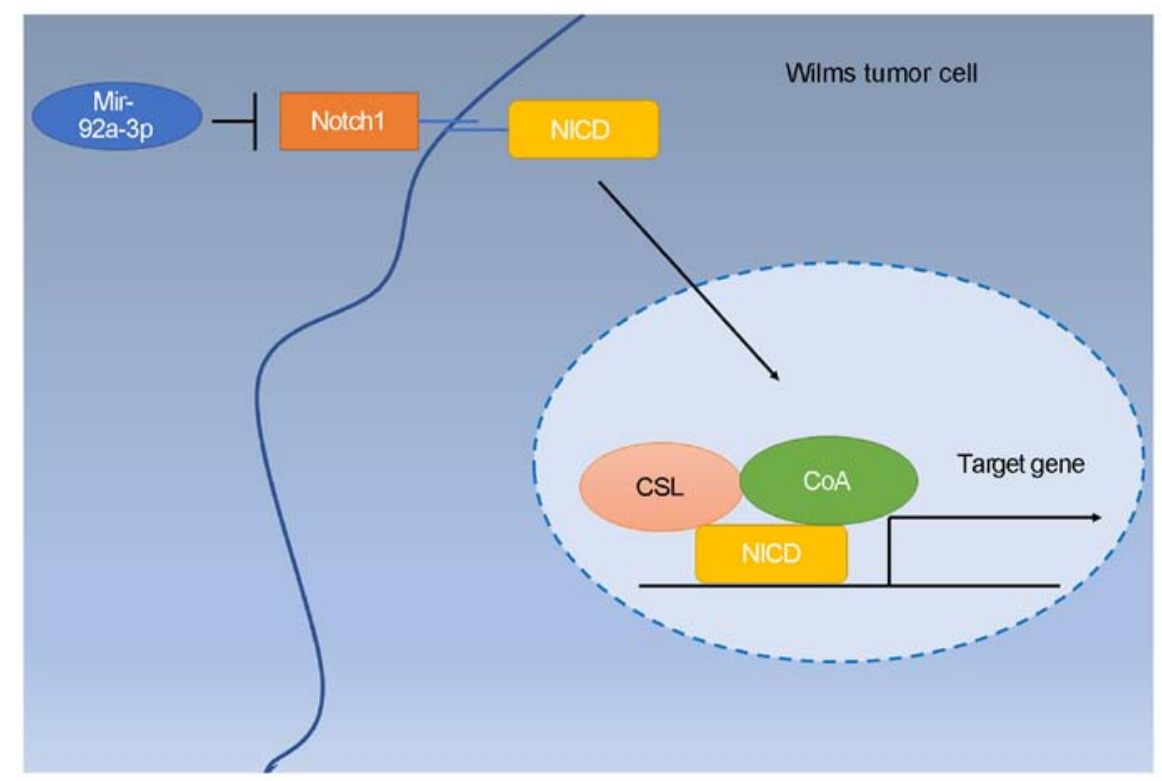

Figure 6. miR-92a-3p blocks the progression of Wilms tumor by targeting NOTCH1. 


\section{Acknowledgements}

The authors thank Mr. zhongmin Li for his technical support.

\section{Funding}

The present study was supported by a grant from the Guangdong Provincial Department of Science and Technology Foundation, P.R. China (no. 2016A020215009).

\section{Availability of data and materials}

All data generated or analyzed during this study are included in this published article.

\section{Authors' contributions}

GL and WJ designed the study; SZ wrote the manuscript, collected clinical information and performed statistical analyses; ZZ and WF assisted with PCR, western blotting and in vitro experiments; LZ assisted with the Dual-Luciferase reporter assays. All authors read and approved the manuscript and agree to be accountable for all aspects of the research in ensuring that the accuracy or integrity of any part of the work are appropriately investigated and resolved.

\section{Ethics approval and consent to participate}

Informed consent was obtained from each patient, and the study protocol and consent procedures were approved by the Ethics Committee of Guangzhou Medical University (Guangzhou, China).

\section{Consent for publication}

Not applicable.

\section{Competing interests}

The authors declare that they have no competing interests.

\section{References}

1. Charlton J, Pavasovic V and Pritchard-Jones K: Biomarkers to detect Wilms tumors in pediatric patients: Where are we now? Future Oncol 11: 2221-2234, 2015.

2. Cone EB, Dalton SS, Van Noord M, Tracy ET, Rice HE and Routh JC: Biomarkers for wilms tumor: A systematic review. J Urol 196: 1530-1535, 2016.

3. Qi C, Hu Y, Yang F, An H, Zhang J, Jin H and Guo F: Preliminary observations regarding the expression of collagen triple helix repeat-containing 1 is an independent prognostic factor for Wilms tumor. J Pediatr Surg 51: 1501-1506, 2016.
4. Brok J, Pritchard-Jones K, Geller JI and Spreafico F: Review of phase I and II trials for Wilms' tumour e Can we optimise the search for novel agents? Eur J Cancer 79: 205-213, 2017.

5. Yu X, Li Z, Chan MT and Wu WK: The roles of microRNAs in Wilms' tumors. Tumor Biol 37: 1445-1450, 2016.

6. Macfarlane LA and Murphy PR: MicroRNA: Biogenesis, function and role in cancer. Curr Genomics 11: 537-561, 2010.

7. Bushati N and Cohen SM: MicroRNA functions. Annu Rev Cell Dev Biol 23: 175-205, 2007.

8. Valinezhad Orang A, Safaralizadeh R and Kazemzadeh-Bavili M: Mechanisms of miRNA-mediated gene regulation from common down-regulation to mRNA-specifc upregulation. Int $\mathrm{J}$ Genomics 2014: 970607, 2014.

9. Tie $\mathrm{J}$ and Fan D: Big roles of microRNAs in tumorigenesis and tumor development. Histol Histopathol 26: 1353-1361, 2011.

10. Hwang HW and Mendell JT: MicroRNAs in cell proliferation, cell death, and tumorigenesis. Br J Cancer 94: 776-780, 2006.

11. Baltimore D, Boldin MP, O'Connell RM, Rao DS and Taganov KD: MicroRNAs: New regulators of immune cell development and function. Nat Immunol 9: 839-845, 2008.

12. Zhang C, Wang C, Chen X, Yang C, Li K, Wang J, Dai J, Hu Z, Zhou X, Chen L, et al: Expression profile of microRNAs in serum: A fingerprint for esophageal squamous cell carcinoma. Clin Chem 56: 1871-1879, 2010.

13. Ma H, Pan JS, Jin LX, Wu J, Ren YD, Chen P, Xiao C and Han J: MicroRNA-17-92 inhibits colorectal cancer progression by targeting angiogenesis. Cancer Lett 376: 293-302, 2016.

14. Zhou P, Ma L, Zhou J, Jiang M, Rao E, Zhao Y and Guo F: miR-17-92 plays an oncogenic role and conveys chemoresistance to cisplatin in human prostate cancer cells. Int $\mathrm{J}$ Oncol 48: 1737-1748, 2016.

15. Song H, Zhang Y, Liu N, Zhao S, Kong Y and Yuan L: miR-92a-3p exerts various effects in glioma and glioma stem-Like cells specifically targeting CDH1/v-catenin and Notch-1/Akt signaling pathways. Int J Mol Sci 17: pii: E1799, 2016.

16. Zheng G, Du L, Yang X, Zhang X, Wang L, Yang Y, Li J and Wang C: Serum microRNA panel as biomarkers for early diagnosis of colorectal adenocarcinoma. Br J Cancer 111: 1985-1992, 2014.

17. Watson JA, Bryan K, Williams R, Popov S, Vujanic G, Coulomb A, Boccon-Gibod L, Graf N, Pritchard-Jones K and O'Sullivan M: MiRNA profiles as a predictor of chemoresponsiveness in Wilms' tumor blastema. PLoS One 8: 53417, 2013.

18. Kopan R and Ilagan MX: The canonical Notch signaling pathway: Unfolding the activation mechanism. Cell 137: 216-233, 2009.

19. Fortini ME: Notch signaling: The core pathway and its posttranslational regulation. Dev Cell 16: 633-647, 2009.

20. Bolos V, Grego-Bessa J and de la Pompa JL: Notch signaling in development and cancer. Endocr Rev 28: 339-363, 2007.

21. Xu P, Qiu M, Zhang Z, Kang C, Jiang R, Jia Z, Wang G, Jiang H and Pu P: The oncogenic roles of Notch1 in astrocytic gliomas in vitro and in vivo. J Neurooncol 97: 41-51, 2010.

22. Boyle SC, Liu Z and Kopan R: Notch signaling is required for the formation of mesangial cells from a stromal mesenchyme precursor during kidney development. Development 141: 345-354, 2014.

(i) (\$) This work is licensed under a Creative Commons Attribution-NonCommercial-NoDerivatives 4.0 International (CC BY-NC-ND 4.0) License. 